\title{
Subwavelength Grating Filters in SOI
}

\author{
Junjia Wang ${ }^{1}$, Ivan Glesk ${ }^{2}$, and Lawrence R. Chen ${ }^{1}$ \\ ${ }^{1}$ Department of Electrical and Computer Engineering, McGill University, Montreal, QC H3A 0E9 Canada \\ ${ }^{2}$ Department of Electronic and Electrical Engineering, University of Strathclyde, Glasgow, G1 1XU, UK
}

\begin{abstract}
We demonstrate subwavelength grating filters in silicon-on-insulator based on Bragg gratings and racetrack resonators. The Bragg grating has a $3 \mathrm{~dB}$ bandwidth $=0.5 \mathrm{~nm}$ and reflectivity $>90 \%$; the resonator has a $3 \mathrm{~dB}$ bandwidth $=1 \mathrm{~nm}$ and extinction ratio $>33$ $\mathrm{dB}$.
\end{abstract}

\section{Introduction}

There is an increasing need for integrated solutions in optical communications, interconnections, and sensing applications [1]. In the past few years, a variety of active and passive devices as well as integrated subsystems in CMOS-compatible silicon photonics platforms have been realized [1,2]. One specific structure that has attracted considerable interest is the subwavelength grating (SWG) [3]. SWG waveguides have the potential for low loss and provide significant flexibility in tailoring the effective index. Indeed, a number of SWG-based building blocks in silicon-on-insulator (SOI) have been developed, including waveguide crossings, bends, couplers, mode transformers, and polarization converters [4]. Recently, we proposed the designs of SWG filters based on Bragg gratings (BGs), microring resonators, and racetrack resonators [5]; however, to date, only microring resonators have been verified experimentally. In this paper, we demonstrate SWG filters in SOI based on BGs and racetrack resonators.

\section{SWG BGs}

Fig. 1a compares the schematic of an SWG waveguide and an SWG BG in SOI. The effective index of the SWG waveguide depends on the duty cycle $f=a / \Lambda$, where $a$ is the width of the high index medium (here $\mathrm{Si}$ ) and $\Lambda$ is the period. An SWG BG can then be realized by interleaving two SWG waveguides with different duty cycles $f_{1}=$ $a_{1} / \Lambda_{1}$ and $f_{2}=a_{2} / \Lambda_{2}$; the SWG BG has a period of $\Lambda_{1}+\Lambda_{2}$. By varying $f_{l}$ and $f_{2}$, we create a periodic variation in the effective index and can thus obtain Bragg reflection. The cross-section of the SWG waveguides used in our experiments is shown in Fig. $1 b$. The width of the silicon layer is $500 \mathrm{~nm}$ and the thickness is $220 \mathrm{~nm}$; it sits on top of a $3 \mu \mathrm{m}$ thick buried oxide (BOX) layer and is covered by an index-matched $2 \mu \mathrm{m}$ thick oxide cladding. We consider SWG waveguides with $\Lambda=280 \mathrm{~nm}$ to obtain a transmission window spanning the C-band and 1,000 periods. Two SWG tapers are used to convert light into (and from) a Bloch mode before (and after) propagating through the SWG waveguide [5]. Vertical grating couplers (VGCs) [6] optimized for TE transmission are used to couple light in and out of the device. A compact Y-branch is used to extract the reflection response of the SWG BGs. The devices were fabricated using electron beam lithography with a single full etch. Figs. $1 c$ and $1 d$ show the SEM image of an SWG BG (before deposition of the top oxide cladding) and the full layout of the device.

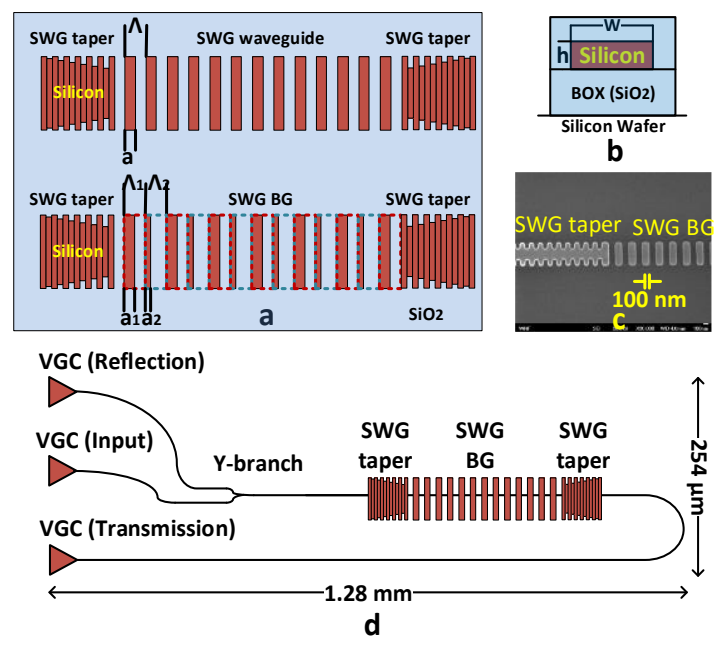

Fig. 1. (a) Top view of an SWG waveguide and an SWG BG, (b) device cross-section, (c) SEM of the fabricated SWG BG prior to top oxide cladding deposition, and (d) full layout.

Fig. $2 a$ shows the measured transmission response of the SWG waveguide without and with an index modulation based on $f_{1}=50 \%$ and $f_{2}=$ $48 \%$. There are no spectral features within the transmission window of the SWG waveguide; on the other hand, the SWG BG exhibits a clear rejection peak at a resonant wavelength of 1546.8 nm. Fig. $2 b$ shows a zoom of the transmission and reflection responses around $1546.8 \mathrm{~nm}$ : the transmission loss is $-12 \mathrm{~dB}$ corresponding to a peak reflectivity of $90.4 \%$; the $3 \mathrm{~dB}$ bandwidth is $0.5 \mathrm{~nm}$. The total fiber-to-fiber loss is typically $15 \mathrm{~dB}$ (input port to reflection port). 
a
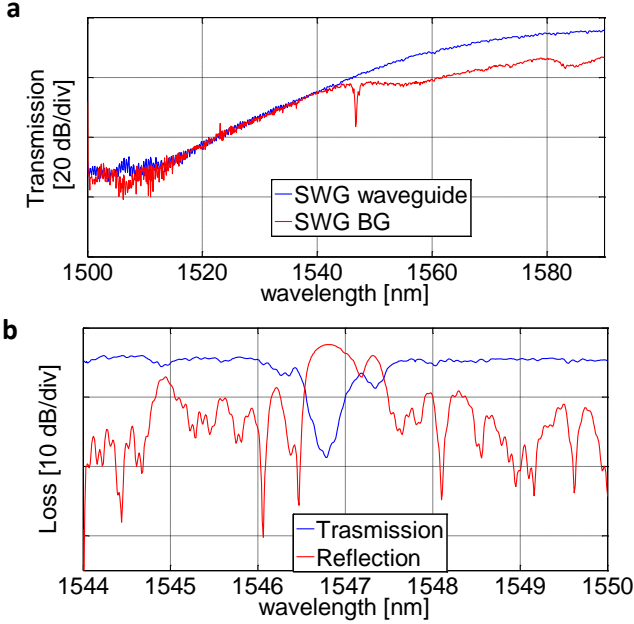

Fig. 2. Measured response of SWG waveguide and SWG BG $\left(f_{l}\right.$ $=50 \%$ and $f_{2}=48 \%$ ).

\section{SWG Racetrack Resonators}

In [5], we demonstrated SWG ring resonators that are under coupled. Racetrack resonators are preferable since the coupling region between the resonator and bus waveguides is longer compared to ring configurations, thereby allowing for greater control over coupling efficiency. Figs. $3 a$ and $3 b$ show the device layout and a zoom of the SWG racetrack resonator. The length of the directional coupling section is $L$ and the separation between the bus and racetrack waveguides (center-to-center) is denoted $g$; these two parameters determine the coupling efficiency and operating condition (under coupled, critically coupled, over coupled) of the resonator. The curved section has a radius $r$ and the total perimeter of the racetrack which determines the free spectral range (FSR) is $2 \pi r+2 L$. We use the same waveguide cross-section and VGC designs as for the SWG BGs. The SWG period is $300 \mathrm{~nm}$ and the duty cycle is $50 \%$ (to obtain a transmission window in the C-band).

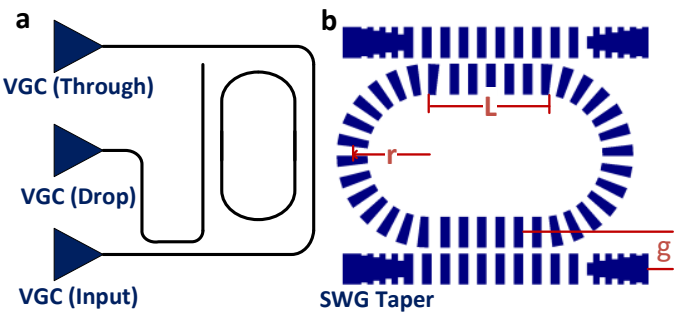

Fig. 3. Schematic of the (a) device layout, and (b) SWG racetrack resonator.

Fig. 4 shows the measured through and drop responses of an SWG racetrack resonator with $r=$ $20 \mu \mathrm{m}, L=40 \mu \mathrm{m}$, and $g=600 \mathrm{~nm}$. The measured FSR is $4.6 \mathrm{~nm}$, which agrees well with the value calculated using the total perimeter of the racetrack and effective waveguide index of 2.47. At 1527.9 $\mathrm{nm}$, near critical coupling is achieved and we obtain an extinction ratio (ER) as high as $33 \mathrm{~dB}$; the $3 \mathrm{~dB}$ bandwidth is $1 \mathrm{~nm}$. The amplitude and ER variations are due to the wavelength dependence of the directional coupling.
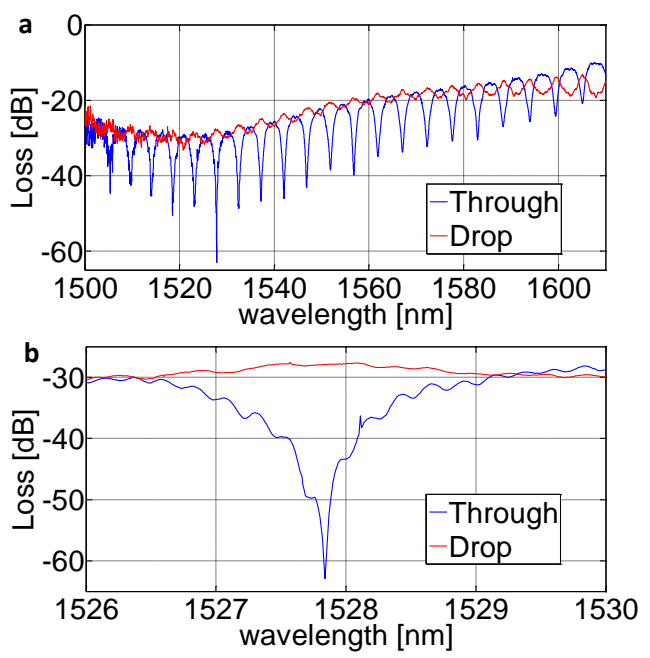

Fig. 4. (a) Measured through and drop responses of a SWG racetrack resonator with $r=20 \mu \mathrm{m}, \mathrm{L}=40 \mu \mathrm{m}$, and $g=600 \mathrm{~nm}$. (b) Zoom in at one resonance at $1527.85 \mathrm{~nm}$.

\section{Conclusions}

We have demonstrated and experimentally verified SWG filters in SOI based on BGs and racetrack resonators. The SWG BG exhibits high reflectivity (> 90\%) and a relatively narrow reflection bandwidth $(0.5 \mathrm{~nm})$. The $\mathrm{SWG}$ racetrack resonator exhibits an extinction ratio as high as $33 \mathrm{~dB}$. Based on the SWG resonators, SWG modulators and higher-order filters can be realized. These filters are compatible with other SWG building blocks and can be used to develop more complex structures with enhanced functionality for applications in communications and sensing.

\section{Acknowledgments}

The devices were fabricated by R. Bojko at the University of Washington Nanofabrication Facility, a member of the NSF National Nantotechnology Infrastructure Network.

\section{References}

1. Soref, R., IEEE Journal of Selected Topics in Quantum Electronics, 2006, 12, (6), pp. 1678-1687.

2. Dong, P. et al, Nanophotonics, 2014, 3, (4-5), pp. 215-228.

3. Bock, P.J. et al, Optics Express, 2010, 18, (19), pp. 2025120262.

4. Halir, R. et al, IEEE Journal of Selected Topics in Quantum Electronics, 2014, 20, (4).

5. Wang, J. et al, Optics Express, 2014, 22, (13), pp. 1533515345.

6. Wang, Y. et al, Optics Express, 2014, 22, (17), pp. 2065220662. 